\title{
Development of a Rehabilitative Training Robot for Both Wrists
}

\author{
Ikuo Yamamoto \\ Graduate School of Engineering \\ Nagasaki University \\ 1-14 Bunkyomachi, \\ Nagasaki 8528521, Japan \\ iyamamoto2011@gmail.com
}

\author{
Naohiro Inagawa \\ Faculty of Engineering \\ Nippon Bunri University \\ 1727 Ichiki, Oita-city, \\ Oita 8700397, Japan \\ inabb1@gmail.com
}

\author{
Kenji Hachisuka, Futoshi Wada \\ and Akiko Hachisuka \\ Department of Rehabilitation Medicine \\ University of Occupational and Environmental Health \\ 1-1 Iseigaoka, Yahatanishi-ku, Kitakyushu, \\ Fukuoka 8078555, Japan \\ kenhachi@med.uoeh-u.ac.jp
}

\begin{abstract}
The authors developed a rehabilitative training robot which detects and analyzes the intention when a person tries to move a wrist with a biological signal, such as muscle action potential, and makes a wrist movement as one's intention. It consists of a grip for wrists, an actuator, a biological signal primary means, biological signal processing part, and a training controller.

Due to its small, compact size, training can be performed in a small space at a hospital or home.

The new system which can conduct both wrists training is successfully developed for practical uses.
\end{abstract}

Index Terms - Compact rehabilitative training robot, Both wrist, Biological signal, Passive mode, Active mode.

\section{INTRODUCTION}

Repetitive training for hemiplegic patients is effective to improve the function of upper and lower extremities and to increase the ranges of motion [1]-[8]. The mild physicallydisabled patients can undergo self-training with tools such as blocks. In contrast, the massive physically-disabled patients generally undergo forced training with training support device. However, a higher effectiveness of training cannot be obtained because patients cannot exercise on their own. Therefore, the effect of the treatment reaches a critical limit. Although occupational therapists can give the patients undivided attention, the numbers of occupational therapists are limited and there is a compelling need to reduce their workload. Although many research results for the rehabilitative training robot have been reported [9]-[14], it is often the case that the system is large, complicated process. Therefore it is difficult to feel free to use the system for patients.

The purpose of this study is the development of a rehabilitative training robot that enables the training of a patient. This training robot detects the myoelectric potential by myoelectric sensors on patient's muscle, and analyzes the patient's intention to flex their wrist [15]. Through this the training robot can provoke training, and the patients can undergo effective training.

A new system based on this function is developed for rehabilitative training of both wrists as shown in Fig.1.

\section{SYSTEM}

A. System Architecture

A configuration diagram is shown in Fig. 2. The rehabilitative training robot consists of the following parts;

1) Grip: The patient holds this grip for undergoing training.

2) Actuator: The training movement is generated by servomotor etc.

3) Biological Signal Detector: The myoelectric potential is detected by myoelectric sensors.

4) Biological Signal Processor: The intention of patients is judged by myoelectric potential.

5) Training Controller: The training movement pattern is generated.

6) Both Wrists System: The training is done using both wrists.

The state transition diagram is shown in Fig. 3.

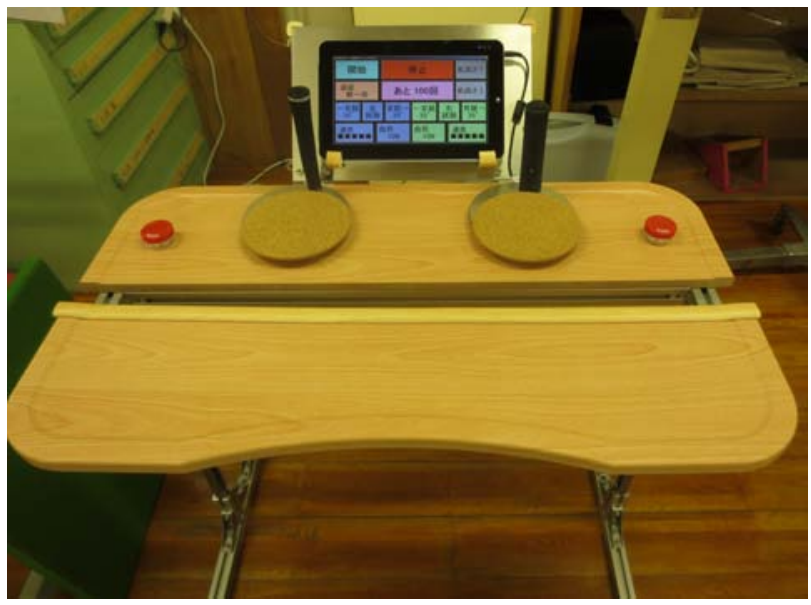

Fig. 1 The new rehabilitative training robot. 


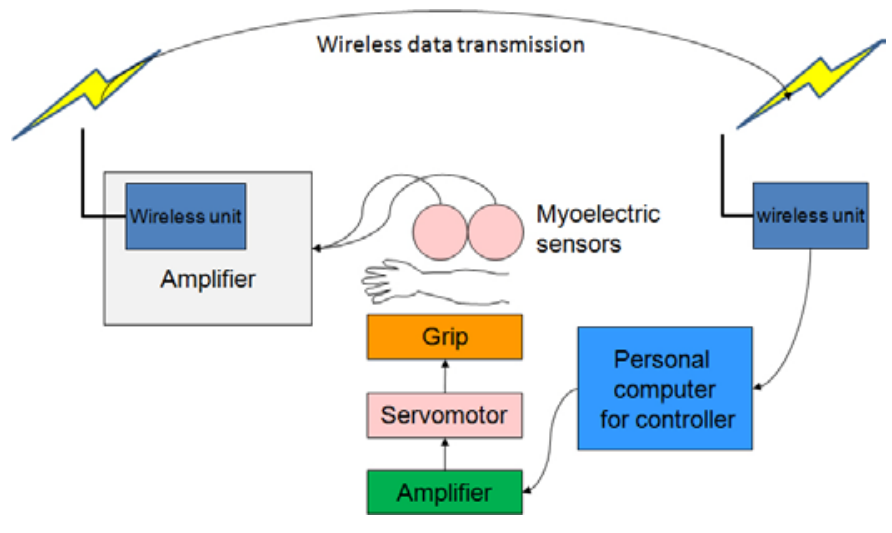

Fig. 2 The configuration diagram of the rehabilitative training robot.

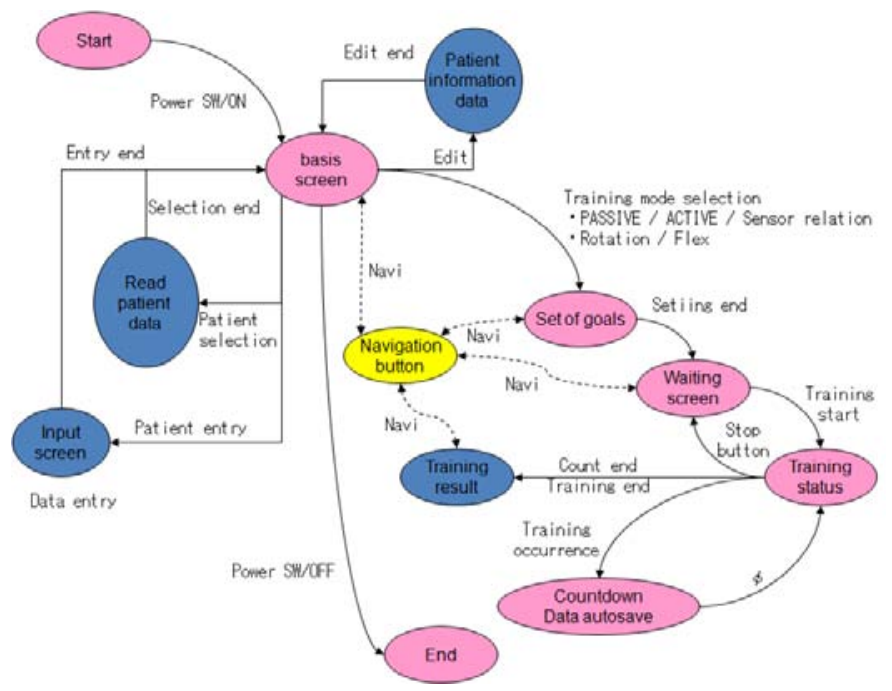

Fig. 3 The state transition diagram of the rehabilitative training robot.

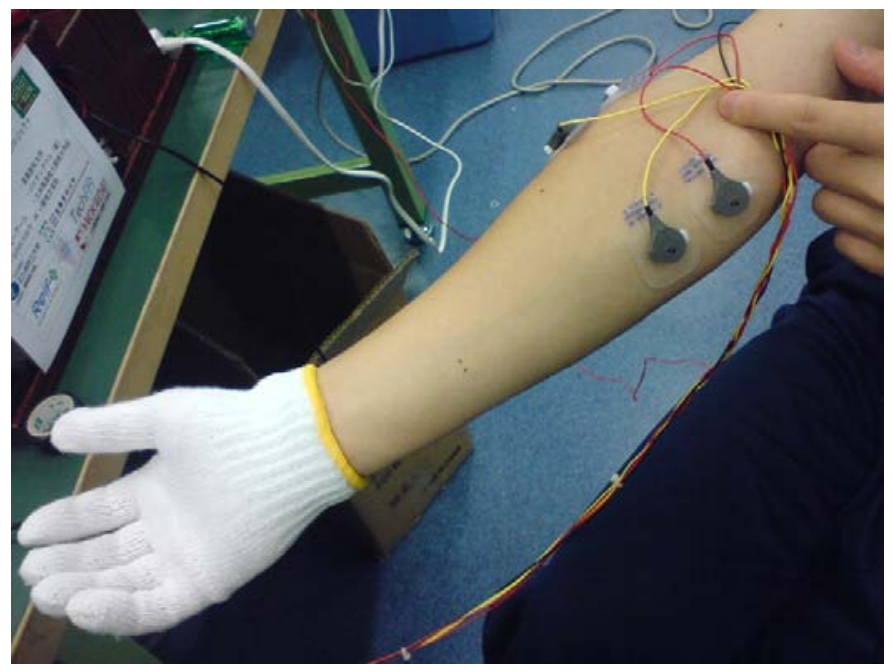

Fig. 4 The myoelectric sensors.

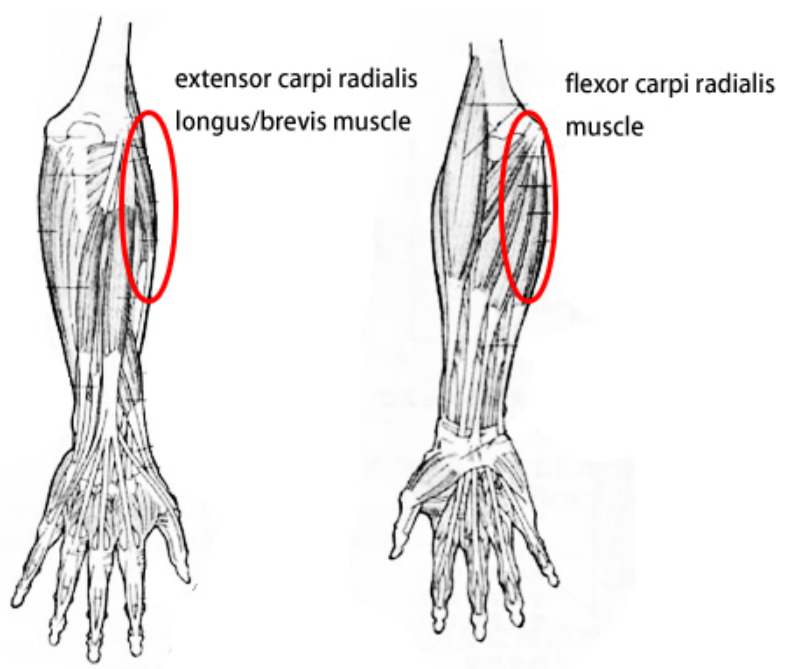

Fig. 5 The extensor carpi radialis longus/brevis muscle and flexor carpi radialis muscle.

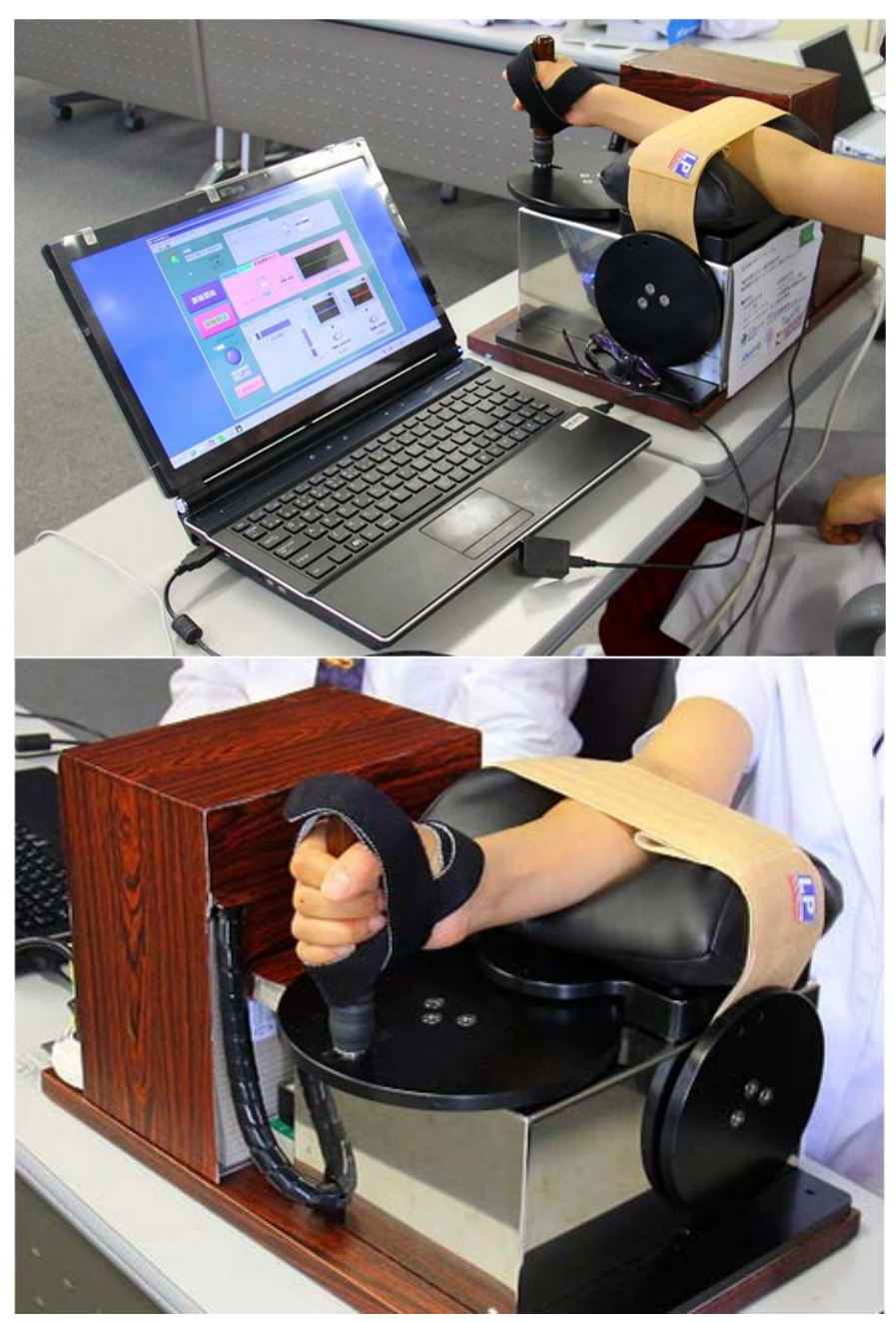

Fig. 6 The appearance of the prototype rehabilitative training robot. 


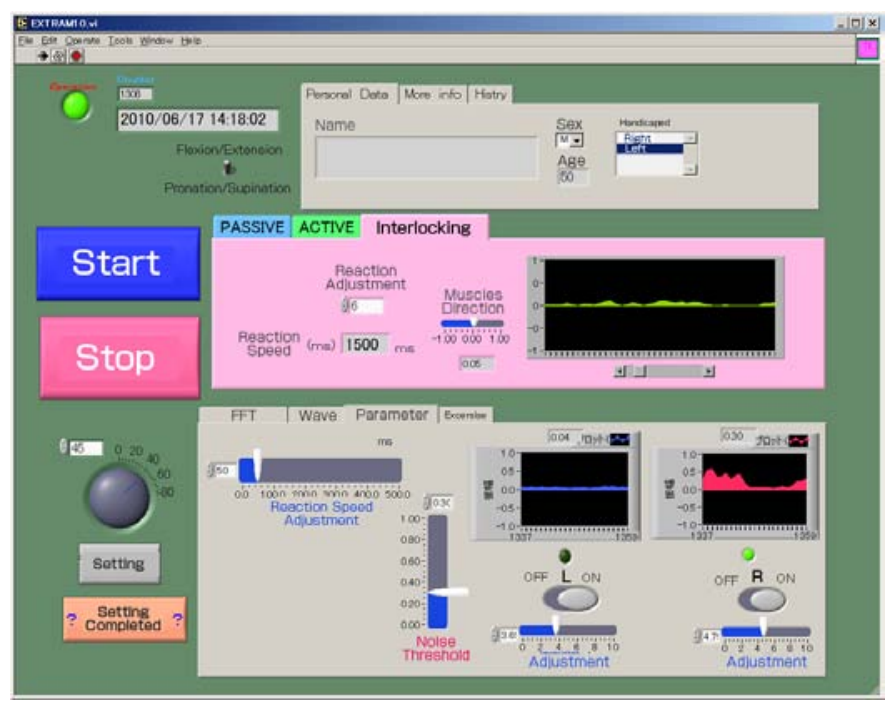

Fig. 7 The advanced settings screen.

TABLE I

Size OF PROTOTYPe REHABILITATIVE TRAINING RoBOT

\begin{tabular}{|c|c|}
\hline & Size $[\mathrm{cm}]$ \\
\hline Width & 50 \\
\hline Height & 34 \\
\hline Depth & 30 \\
\hline
\end{tabular}

\section{B. Myoelectric Sensors}

The patient's intention to flex their wrist is transmitted to the muscles of the wrist through nerves from the brain as myoelectric potential. The myoelectric potential is detected by myoelectric sensors. The myoelectric sensors used in our system are shown in Fig. 4. The four myoelectric sensors are worn on the extensor carpi radialis longus/brevis muscle and flexor carpi radialis muscle as shown in Fig. 5. The myoelectric potential which is detected by myoelectric sensors is amplified and is analyzed by software on a personal computer. The actuator is moved based on the analysis result. The developed rehabilitative training robot system supports rehabilitative trainings based on the patient's intention to flex their wrist.

\section{Passive and Active Mode}

The developed rehabilitative training robot is equipped with active mode in addition to passive mode.

1) Passive Mode: Although the robot moves according to the flex of patient, the robot is only to assist the force.

2) Active Mode: The myoelectric sensors detect the patient's intention to flex their wrist, and the robot takes the lead in moving.

The patients are able to undergo not only passive training but also active training with the use of the passive and active modes. Therefore the training effectiveness can be expected to increase.

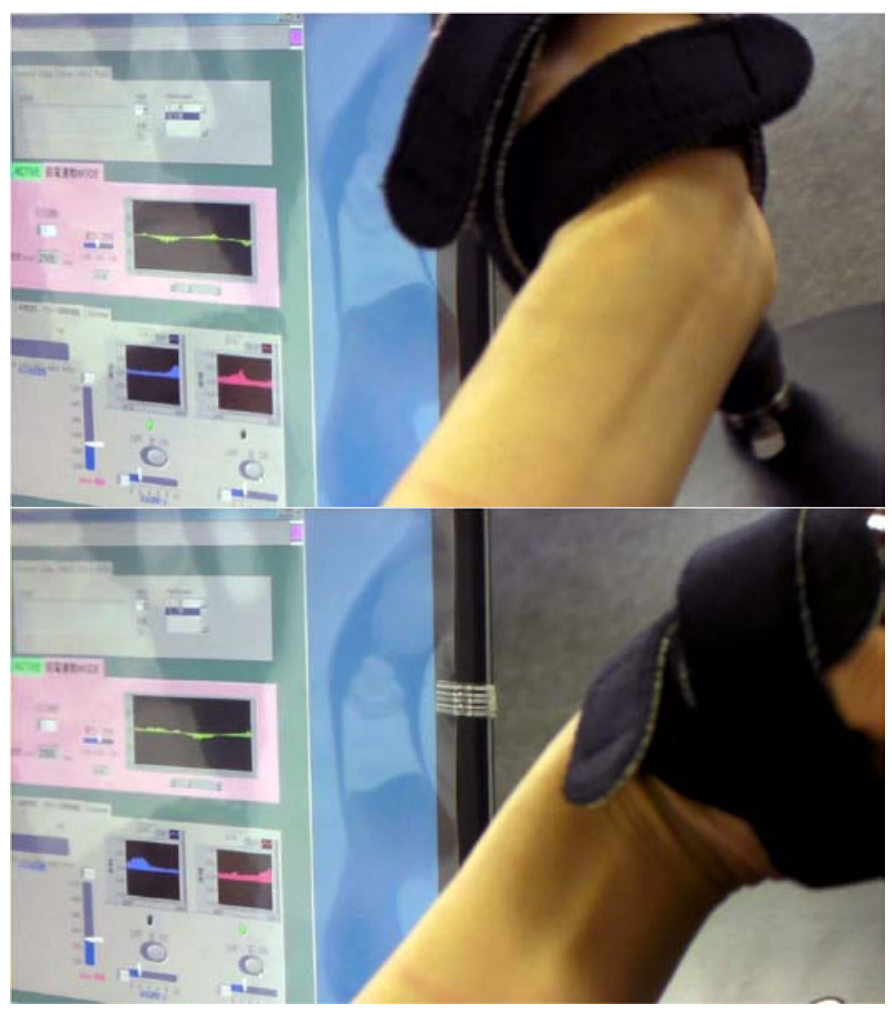

Fig. 8 The developed rehabilitation robot in an excited condition.

\section{Appearance of Robot}

The developed rehabilitative training robot is easy to carry because of its compact size. The training can therefore be performed in a small space at the hospital or home. Table 1 shows the size of the prototype rehabilitative training robot. Fig. 6 shows the appearance of the prototype rehabilitative training robot.

\section{EXPERIMENTAL TRIAL}

Fig. 7 shows an advanced settings screen of the developed rehabilitative training robot. The middle graphic (green line) represents the myoelectric potential which was detected by myoelectric sensors. The myoelectric potential data is analyzed and the actuator (servomotor) is moved based on the analysis result. Fig. 8 shows the developed rehabilitative training robot in an excited condition.

\section{FEEDBACK FROM EXPERIMENTAL TRIAL}

Fig. 9 shows a new display by feedback for training. It is user-friendly for patients with easily recognizable touch panels which should provide little chance for operational user error. 


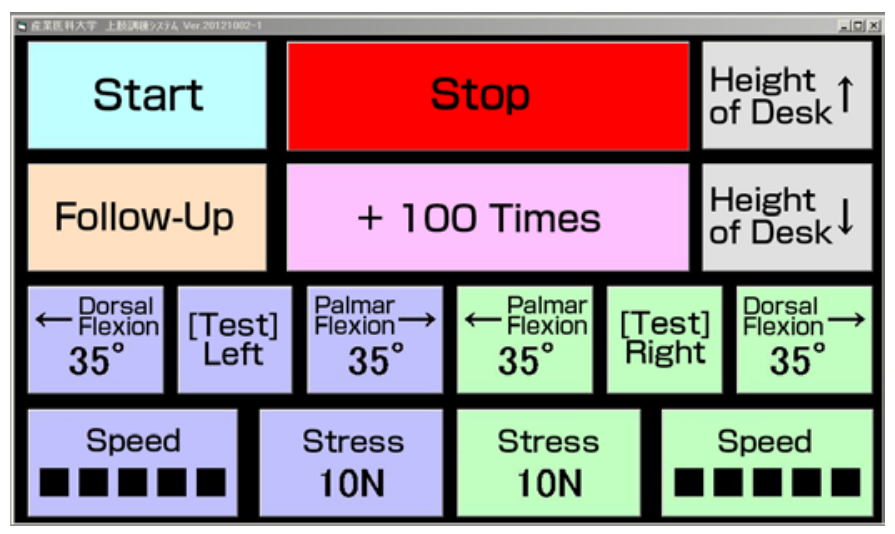

Fig. 9 The user-friendly screen of the new rehabilitative training robot.

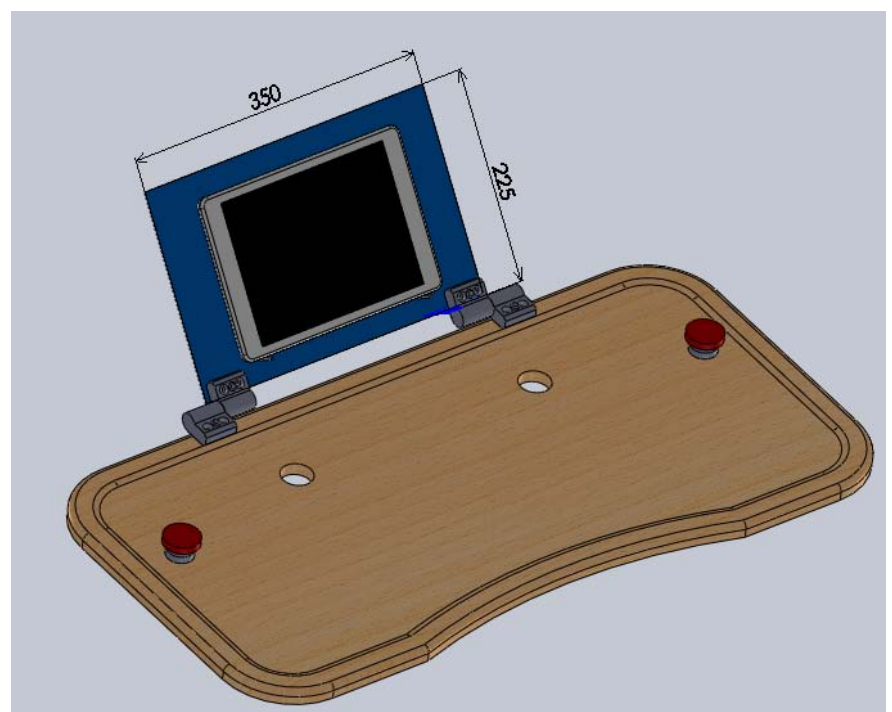

Fig. 10 The CAD design of the new rehabilitative training robot.

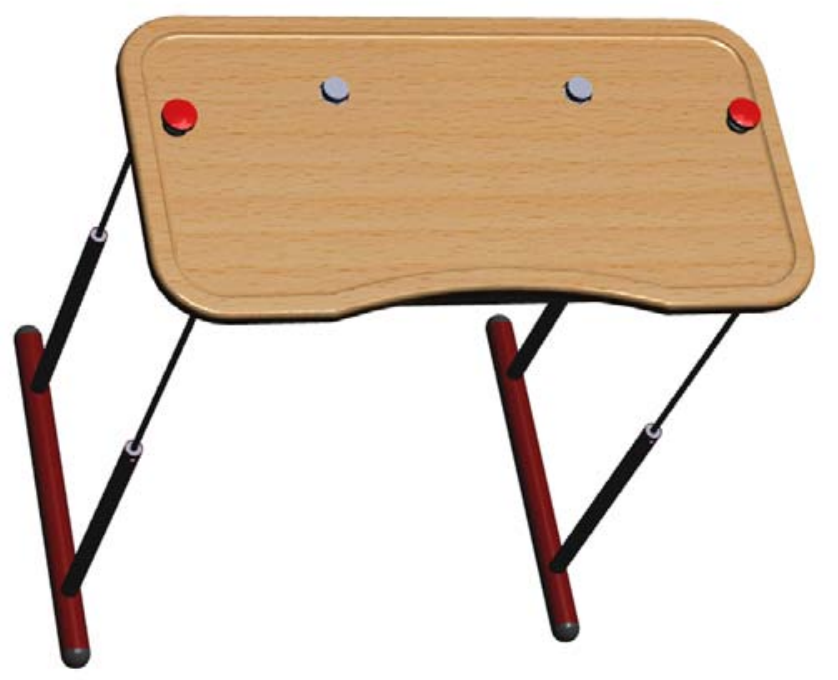

Fig. 11 The CAD design of the new rehabilitative training robot for hospital use.

\section{ReHABILITATIVE TRAining RoBot FOR PATIENTS}

Fig. 10 shows a CAD design of the rehabilitative training robot for patients' use. Fig. 11 shows a CAD design of the rehabilitative training robot for hospital use. The height of the robot is changeable.

\section{CONCLUSIONS}

The authors reported a simple rehabilitative training robot which detects and analyzes the intention of a patient, and assists patient's wrist movement. It consists of a grip for the wrist, actuator, biological signal processor, and controller. Motor unit action potential is detected as a biological signal, and the signal is processed to assist wrist movement. Although there are many rehabilitative training robots developed, most of the robots are complicated, expensive, and difficult to use. Because this rehabilitative training robot is a small sized device and is easily operated by a patient and family members, it will be useful for home use as well as hospital use.

\section{ACKNOWLEDGMENT}

The authors would like to express their sincere gratitude to ETOU Inc. for manufacturing the robot system.

\section{REFERENCES}

[1] S. Saeki, Y. Matsushima, K. Hachisuka, "Cortical activation during robotic therapy for a severely affected arm in a chronic stroke patient: a case report,” J. UOEH, vol. 30, no. 2, pp. 159-165, 2008.

[2] G.B. Prange, M.J.A. Jannink, C.G.M. Groothuis-Oudshoon, H.J. Hermens, M.J. IJzerman, "Systematic review of the effect of robot-aided thrapy on recovery of the hemiparetic arm after stroke," J. Rehabil Res Dev, vol. 43, pp. 171-184, 2006.

[3] H.I. Krebs, N. Hogan, B.T. Volpe, M.L. Aisen, L. Edelstein, C. Diels, "Overview of clinical trials with MIT-MANUS: Arobot-aided neurorehabilitation facility,” Technol. Health Care, vol. 7, pp. 419-423, 1999.

[4] C.G. Burgur, P.S. Lum, P.C. Shor, H.F. Machiel Van der Loos, "Development of robots for rehabilitation therapy: The Palo Alto VA/Standard experience,” J. Rehabil. Res. Dev., vol. 37, pp. 376-388, 2000.

[5] S. Masiero, A. Celia, G. Rosati, M. Armani, "Robotic-assisted rehabilitation of the upper limb after acute stroke," Arch. Phys. Med. Rehabil., vol. 88, pp. 142-149, 2007.

[6] S. Hesse, G. Schulte-Tiggers, M. Konrad, M.A. Bardeleben, C. Werner, "Robot-assisted arm trainer for the passive and active practice of bilateral forearm and wrist movements in hemiparetic subjects," Arch. Phys. Med. Rehabil., vol. 84, pp. 915-920, 2003.

[7] S. Hesse, C. Werner, M. Pohl, S. Rueckriem, J. Mehrholz, M.L. Lingnau, "Computerized arm training improves the motor control of the severely affected arm after stroke: a single-blinded randomized trial in two centers," Stroke, vol. 36, pp. 1960-1966, 2005.

[8] M. Ferraro, J.J. Palazzolo, J. Krol, H.I. Krebs, N. Hogan, B.T. Volpe, "Robot-aided sensorimoter arm training improves outcome in patients with chronic stroke,” Neurology, vol. 61, pp. 1604-1607, 2003.

[9] Guan De Lee, Wei-Wen Wang, Kai-Wen Lee, Sheng-Yen Lin, Li-Chen Fu, Jin-Shin S. Lai, Wen-Shiang Chen, Jer-Junn J. Luh, "Arm exoskeleton rehabilitation robot with assistive system for patient after stroke," Proc. of ICCAS, pp. 1943-1948, 2012.

[10] Hong-Bo B. Wang, Ning Du, Huina Hou, Jiangshun Zhao, Hongnain N. $\mathrm{Yu}$, "An upper limb rehabilitation robot based on 3-RRC parallel mechanism," Proc. of ICAMechS, pp. 668-673, 2012. 
[11] Xikai Tu, Jian Huang, Liguo Yu, Qi Xu, Jiping He, "Design of a wearable rehabilitation robot integrated with functional electrical stimulation," Proc. of IEEE RAS \& EMBS BioRob, pp. 1555-1560, 2012.

[12] Lei Shi, Kun Jiang, Qiang Wang, Zhen Liu, "A New Method of Quantitative Rehabilitation Rating in Robot-Assisted Recovery System," Proc. of ICINIS, pp. 278-281, 2012.

[13] Yongsuk Kang, Doyoung Jeon, "Rehabilitation robot control using the VSD method," Proc. of SII, pp. 192-197, 2012.

[14] Chao Lv, Le Xie, Wei Shao, Hai-long Yu, Yuan Wang, Jin-wu Wang, Ning Nan, "Development of a Rehabilitation Robot for Upper-Limb Movements," Intelligent Robotics and Applications, Lecture Notes in Computer Science, Vol.7507, pp. 96-102, 2012.

[15] I. Yamamoto, N. Inagawa, K. Hachisuka, F. Oda, Y. Nakanishi, "Development of Compact Rehabilitation Robot for a Wrist Using Biological Signal,” Proc. of IEEE CME, pp. 557-560, 2012. 